\title{
XRP44X Enhances the Cytotoxic Activity of Natural Killer Cells by Activating the c-JUN N-Terminal Kinase Signaling Pathway
}

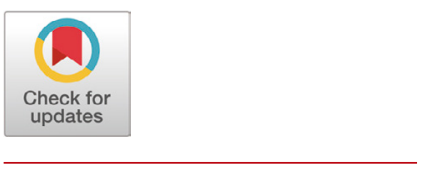

Received: January 14, 2020

Revised: January 17, 2020

Accepted: January 28, 2020

${ }^{+}$Corresponding author Kyung-Soon Park

Dept. of Biomedical Science, College of Life Science, CHA University,

Seongnam 13488, Korea.

Tel: +82-31-881-7144

Fax: +82-31-881-7249

E-mail:kspark@cha.ac.kr

Copyright @ 2020 The Korean Society of Developmental Biology.

This is an Open Access article distributed under the terms of the Creative Commons Attribution Non-Commercial License (http://creativecommons.org/licenses/ by-nc/4.0/) which permits unrestricted non-commercial use, distribution, and reproduction in any medium, provided the original work is properly cited.

ORCID

Kwang-Soo Kim

https://orcid.org/0000-0001-7535-7088 Kyung-Soon Park

https://orcid.org/0000-0002-0615-4313

Conflict of interests

The authors declare no potential conflict of interest.

Acknowledgements

This research was supported by the Ministry of Education, Science, and Technology (NRF-2019R1A2C1003581) and by the Basic Science Research Program through the National Research Foundation (NRF) of Korea, which is funded by the Ministry of Education (2019R1A6A1A03032888).

Authors' contributions Conceptualization: Park KS.

Data curation: Kim KS

Formal analysis: Park KS, Kim KS.

\author{
Kwang-Soo Kim and ${ }^{\dagger}$ Kyung-Soon Park \\ Dept. of Biomedical Science, College of Life Science, CHA University, Seongnam 13488, Korea
}

\section{Abstract}

Natural killer (NK) cells are innate lymphocytes that play an essential role in preventing cancer development by performing immune surveillance to eradicate abnormal cells. Since ex vivo expanded NK cells have cytotoxic activity against various cancers, including breast cancers, their clinical potential as immune-oncogenic therapeutics has been widely investigated. Here, we report that the pyrazole chemical XRP44X, an inhibitor of Ras/ ERK activation of ELK3, stimulates NK-92MI cells to enhance cytotoxic activity against breast cancer cells. Under XRP44X stimulation, NK cells did not show notable apoptosis or impaired cell cycle progression. We demonstrated that XRP44X enhanced interferon gamma expression in NK-92Ml cells. We also elucidated that potentiation of the cytotoxic activity of NK-92MI cells by XRP44X is induced by activation of the c-JUN N-terminal kinase (JNK) signaling pathway. Our data provide insight into the evaluation of XRP44X as an immune stimulant and that XRP44X is a potential candidate compound for the therapeutic development of NK cells.

Keywords: Natural killer cells, NK-92MI, XRP44X, MDA-MB231, c-JUN N-terminal kinase (JNK)

\section{INTRODUCTION}

Natural killer (NK) cells are cytotoxic lymphocytes that are capable of killing virus-infected cells and cancer cells. Unlike CD8+ T cells, which have cytotoxic activity after adaptive differentiation by the recognition of antigen peptides bound to major histocompatibility complex (MHC)-I molecules, NK cells do not require a differentiation process to eliminate contacted target cells and display rapid cellmediated cytotoxicity. Therefore, NK cells are regarded as immune cells that function as the first line of defense against cancer cells and viral infections (Janeway \& Medzhitov, 2002). The cytotoxic ability of NK cells to recognize and kill target cells is controlled by the integration of signals that result from various combinatory contacts between cell surface receptors on NK cells and matching ligands on target cells. NKG2D is an activating receptor on NK cells (Bauer et al., 1999), and stimulation of NK cells by NKG2D ligands induces activation of various signals, including the phosphatidylinositol 3-kinase (PI3K) and c-JUN N-terminal kinase (JNK) MAP kinase pathways (Sutherland et al., 2002; Li et 
Methodology: Park KS, Kim KS. Investigation: Park KS.

Writing - original draft: Kim KS.

Writing - review \& editing: Park KS, Kim KS.

Ethics approval

This article does not require IRB/IACUC approval because there are no human and animal participants. al., 2008). In particular, activation of JNK kinase is required for the movement of the microtubule organizing center (MTOC) and granule polarization in activated NK cell cytotoxicity ( $\mathrm{Li}$ et al., 2008).

In addition to target recognition by receptor-ligand interactions, the cytotoxicity of NK cells is modulated by other factors, including interleukins (IL-12 and IL-15, for example) (Huntington., 2014; Sim et al., 2014; Ge et al., 2017). NK cells are also activated by phorbol 12-myristate 13-acetate (PMA) and the ionophore ionomycin (ION), which lead to increased NK cell degranulation (Romera-Cárdenas et al., 2016). Many compounds have also been identified as activators of the protein kinase $\mathrm{C}(\mathrm{PKC})$ signaling pathway, which is a major lytic signaling pathway in NK cells (Rana \& Whalen., 2015). Stimulation of NK cells by exogenous factors generally results in increased killing of target cells, increased levels of degranulation or increased interferon- $\gamma$ production.

ELK3 is a proto-oncogenic transcription factor of the ETS family and is a therapeutic target in various cancers, including breast cancer and liver cancer (Kong et al., 2016; Lee et al., 2017). $\mathrm{XRP} 44 \mathrm{X}$ is a pyrazole chemical that inhibits the transcriptional activity of ELK3. XRP44X inhibits Ras signaling-mediated phosphorylation of ELK3, and the application of XRP44X results in various cellular effects, including G2-M cell cycle arrest and suppression of angiogenesis (Wasylyk et al., 2008). In addition, XRP44X acts as a microtubule depolymerization agent by activating the JNK pathway (Chen et al., 2012). Administration of XRP44X to preclinical mouse tumor model resulted in the inhibition of tumor growth and metastasis (Semenchenko et al., 2016).

Since the ETS family transcription factor ETS1 regulates the expression of signaling molecules that are essential for NK cell activation and $E t s 1^{-/}$NK cells are in a chronically activated state (Ramirez et al., 2012), we examined whether the application of XRP44X, an inhibitor of the ETS family gene ELK3, modulated the cytotoxic activity of NK cells.

We found that XRP44X stimulated NK cells to enhance cytotoxic activity against breast cancer cells. In the presence of XRP44X, NK cells did not show notable apoptosis or impaired cell cycle progression. We demonstrated that XRP44X enhanced the cytotoxicity of NK cells through stimulation of interferon gamma expression and activation of the JNK signaling pathway. Our data suggest that XRP44X has the potential to be applied in developing NK cells as an immuneoncogenic therapeutic.

\section{MATERIALS AND METHODS}

\section{Cells and reagents}

NK-92MI and MDA-MB231 cells were purchased from the American Type Culture Collection (ATCC, Manassas, VA, USA). The human NK cell line NK-92MI was cultured in Minimum Essential Medium alpha (MEM- $\alpha$, Invitrogen, Carlsbad, CA, USA) supplemented with $2 \mathrm{mM}$ L-glutamine (Invitrogen), $1.5 \mathrm{~g} / \mathrm{L}$ sodium bicarbonate (Sigma-Aldrich, St. Louis, MO, USA), $0.2 \mathrm{mM}$ myo-inositol (Sigma-Aldrich), $0.1 \mathrm{mM}$ 2-mercaptoethanol (Invitrogen), $0.02 \mathrm{mM}$ folic acid (Sigma-Aldrich), 12.5\% fetal bovine serum (Invitrogen) and 1\% penicillin/streptomycin (Invitrogen). XRP44X was purchased from Sigma-Aldrich and dissolved in dimethyl sulfoxide (DMSO, Invitrogen).

\section{Apoptosis assay}

To detect apoptosis, NK-92MI cells $\left(5 \times 10^{5}\right)$ were cultured in a 12 -well cell culture plate and treated with XRP44X. After an additional $48 \mathrm{hr}$ of culture, the cells were harvested and resuspended in an appropriate volume of cold phosphate-buffered saline (PBS, Invitrogen) for analysis. Treated 
cells were stained by using an annexin V-FITC/PI apoptosis kit (Invitrogen) to monitor apoptotic cells. The collected cells were obtained and analyzed using a CytoFLEX flow cytometer (Beckman Coulter, Brea, CA, USA).

\section{Cell cycle analysis}

NK-92MI cells were treated with XRP44X for $48 \mathrm{hr}$, harvested, washed, resuspended in 70\% ethanol and stored at $4{ }^{\circ} \mathrm{C}$ overnight. Then, the cells were suspended in cold PBS. Subsequently, the cells were incubated with $0.1 \mathrm{mg} / \mathrm{mL}$ RNase I and $50 \mathrm{mg} / \mathrm{mL}$ PI at $37^{\circ} \mathrm{C}$ for $30 \mathrm{~min}$. The cell cycle was then detected with a CytoFLEX flow cytometer (Beckman Coulter).

\section{Cytotoxicity assay}

MDA-MB231 cells $\left(1 \times 10^{5}\right)$ were prestained with CellTrace CFSE (Invitrogen) and cocultured with NK-92MI cells at a 10:1 effector:target (E:T) ratio. After $4 \mathrm{hr}$ of coincubation, all cells were harvested and stained with 7-AAD (Invitrogen) for live/dead discrimination. The samples were analyzed on a CytoFLEX flow cytometer (Beckman Coulter). Triplicate experiments were performed.

The cytotoxicity of NK cells was also determined using a CytoTox-Glo ${ }^{\mathrm{TM}}$ cytotoxicity assay (Promega, Madison, WI, USA) according to the manufacturer's instructions. After $4 \mathrm{hr}$ of coincubation of NK-92MI and MDA-MB231 cells, $50 \mu \mathrm{L}$ of CytoTox-Glo ${ }^{\mathrm{TM}}$ cytotoxicity assay reagent was added to the reactions, and the luminescent signal that reflects the cytotoxicity was measured using a SpectraMax L microplate reader (Molecular Devices, Sunnyvale, CA, USA). Cytotoxicity was calculated by dividing the luminescent dead-cell signal by the total cell luminescence value.

\section{RNA extraction and quantitative RT-PCR}

Total RNA from NK-92MI cells was extracted using TRIzol reagent (Invitrogen). One microgram of total RNA was treated with RNase-free DNase I (Invitrogen), and cDNA was prepared using the SuperScript II First-Strand Synthesis System (Invitrogen). Quantitative RTPCR was performed with iQ SYBR Green PCR Mastermix (Bio-Rad, Hercules, CA, USA).

\section{Protein extraction and immunoblot analysis}

For protein analysis, cells were washed twice with cold PBS (Invitrogen) and lysed in cell lysis buffer (Cell Signaling Technology, Danvers, MA, USA). Total cell extracts were resolved by sodium dodecyl sulfate-polyacrylamide gel electrophoresis, transferred to polyvinylidene fluoride (PVDF) membranes (Bio-Rad), and blotted with antibodies against GAPDH, phospho-JNK, JNK, phospho-NF-kB, NF-B, phospho-p38, p38, phospho-AKT, AKT, phospho-ERK1/2, ERK1/2 (Cell Signaling Technology), ELK3 (Novus, Centennial, CO, USA), and phospho-ELK3 (GeneTex, Irvine, CA, USA). Immunoreactivity was detected with enhanced chemiluminescence (Thermo Fisher Scientific, Rochester, NY, USA).

\section{Enzyme-linked immunosorbent assay}

To determine the level of IFN- $\gamma$ in XRP44X-treated NK-92MI cell culture media, ELISA was performed. All reagents were obtained from BD (Franklin Lakes, NJ, USA). Immunoplates were coated overnight at $4{ }^{\circ} \mathrm{C}$ with coating antibodies and buffer. Plates and reagents were brought to room temperature, and the plate was washed three times with washing buffer. The plates were blocked for $1 \mathrm{hr}$ at room temperature with $100 \mu \mathrm{L}$ blocking buffer. Then, the samples and standards were added to each well for $2 \mathrm{hr}$ at room temperature. After washing, $100 \mu \mathrm{L}$ of detection antibody 
solution was added to each well for $1 \mathrm{hr}$ at room temperature. For color development, $50 \mu \mathrm{L}$ TMB was added to each well. The plates were incubated for $30 \mathrm{~min}$ in the dark before the reaction was stopped by adding $50 \mu \mathrm{L}$ of stop solution to each well. The absorbance was mEeasured at $450 \mathrm{~nm}$ using a microplate reader.

\section{Statistical analysis}

Samples were analyzed with Student's $t$-test or ANOVA with Duncan's multiple range procedure for multiple comparisons. All statistical analyses were performed using GraphPad Prism 6.0 software (GraphPad Software, USA) or the SigmaPlot 11.2 program (Systat Software, USA). The error bars represent the standard errors from three independent experiments, which were each performed using triplicate samples. $p$-values less than 0.05 were considered statistically significant.

\section{RESULTS}

To determine the concentration of XRP44X that was not cytotoxic, we first examined the effect of various concentrations of XRP44X on the cell cycle of the human NK cell line NK-92MI. As shown in Fig. 1A, $0.8 \mu \mathrm{M}$ XRP44X induced a slight increase in the G2/M population, which is consistent with the activity of XRP44X in MCF-7 breast cancer cells (Chen et al., 2012).

Although $0.8 \mu \mathrm{M}$ XRP44X affected the cell morphology of MDA-MB231 cells, NK cell morphology was not affected by the same concentration of XRP44X (data not shown). To further confirm whether cell viability was affected, we performed annexin V/PI staining analysis. Consistent with the cell cycle analysis, $0.8 \mu \mathrm{M}$ XRP44X did not increase the dead cell population of NK-92MI cells (Fig. 1B). Based on these results, we concluded that XRP44X does not have toxic effects on NK-92MI cells up to a concentration of $0.8 \mu \mathrm{M}$, and we further analyzed the effect of XRP44X on the cytotoxic activity of NK cells against cancer cells at a concentration of $0.8 \mu \mathrm{M}$.

To analyze whether XRP44X increases the cytotoxic activity of NK-92MI cells toward the triple negative breast cancer cell line MDA-MB231, we pretreated NK-92MI cells with XRP44X for $48 \mathrm{hrs}$ and then cocultured the cells with MDA-MB231 cells for $4 \mathrm{hrs}$. When the cytotoxicity of NK-92MI was quantified using a CytoTox $\mathrm{Glo}^{\mathrm{TM}}$ cytotoxicity assay that selectively measures dead-cell protease activity, it was clearly revealed that the cytotoxic activity of NK-92MI cells against MDA-MB231 cells gradually increased in a XRP44X concentration-dependent manner

A

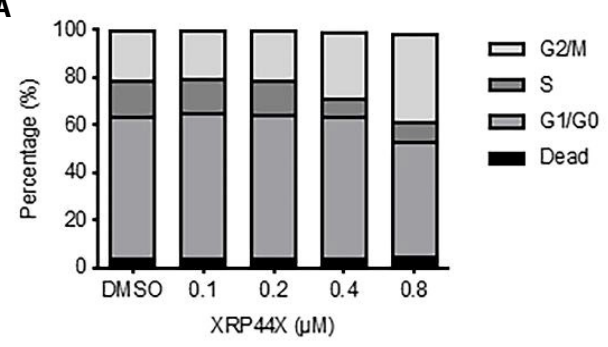

B

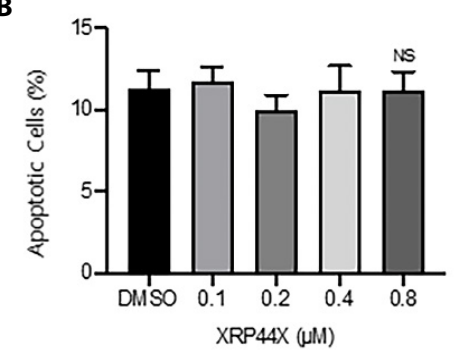

Fig. 1. The effect of XRP44X on the cell cycle and viability of NK-92MI cells. The effect of XRP44X on (A) the cell cycle and (B) apoptosis of NK-92Ml cells. The indicated concentrations of XRP44X were added to NK-92Ml cells for $48 \mathrm{hr}$, and then the cell cycle was analyzed with a CytoFLEX flow cytometer. To monitor apoptotic cell death, XRP44X-treated cells were stained with annexin V-FITC/PI before FACS analysis. The error bars represent the standard errors from three independent experiments, which were each performed using triplicate samples. NS, not significant (Student's $t$-test). 
(Fig. 2A). The effect of XRP44X on the cytotoxicity of NK-92MI was further supported by flow cytometry analysis with CFSE/7-AAD staining. Similar to the dead cell protease analysis results, the target cell lysis ability of NK-92MI was increased by treatment with $0.8 \mu \mathrm{M}$ XRP44X (Fig. 2B). By analyzing the cytotoxicity of NK cells that were pretreated with XRP44X for various times, we identified that the effect of XRP44X on NK-92MI cells gradually increased depending on the pretreatment time, and we also revealed that $0.8 \mu \mathrm{M}$ XRP44X increased NK cell activity with as little as $6 \mathrm{hr}$ of pretreatment (Fig. 2C).

Since NK cells exhibit a cytotoxic immune response by producing IFN- $\gamma$, we next examined whether XRP44X stimulates NK cells to produce increased amounts of IFN- $\gamma$. As expected, IFN- $\gamma$ transcripts were significantly enhanced by treatment with $0.4 \mu \mathrm{M}$ and $0.8 \mu \mathrm{M}$ XRP44X (Fig. 3A). Consistently, secreted IFN- $\gamma$ in NK cell culture media was increased in a XRP44X concentration-dependent manner (Fig. 3B). The amount of secreted IFN- $\gamma$ was further increased when XRP44X-treated NK-92MI cells were cocultured with MDA-MB231 cells (Fig. 3C). These results suggest that the mechanism by which XRP44X increases the cytotoxic activity of NK-92MI cells toward MDA-MB231 cells is by stimulating IFN $-\gamma$ expression and secretion.

The mitogen-activated protein kinase (MAPK) signaling pathway is reported to be activated during NKG2D-mediated NK cell cytotoxicity (Li et al., 2008). To examine whether the MAPK signaling pathway is activated by XRP44X treatment, we examined the phosphorylation status of c-JUN N-terminal kinase (JNK), p38 and extracellular signal-regulated protein kinase (ERK)

A

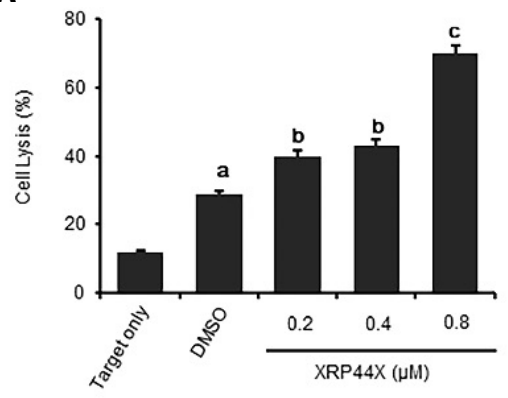

B

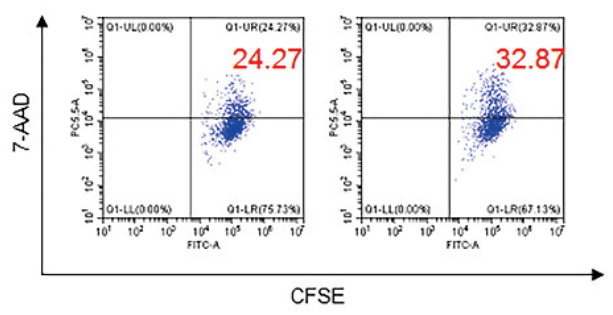

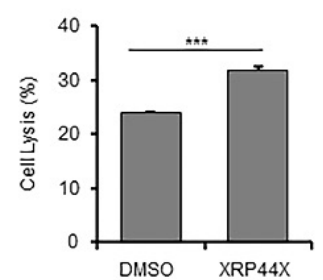

C

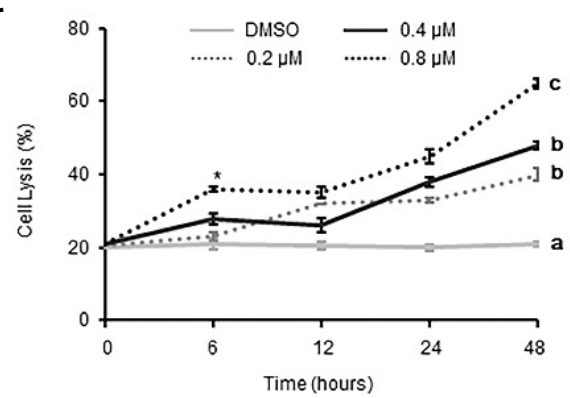

Fig. 2. XRP44X increases the cytotoxic activity of NK cells toward MDA-MB231 cells. (A) The effect of XRP44X on the cytotoxicity of NK-92MI cells was analyzed by a CytoTox Glo ${ }^{\mathrm{TM}}$ cytotoxicity assay. The indicated concentration of XRP44X was added to NK-92-MI cells for 48 hrs, and then the cells were coincubated with MDA-MB231 cells (E:T ratio=10:1) for 4 hrs. (B) NK-92Ml cells were treated with $0.8 \mu \mathrm{M}$ XRP44X for 48 hrs, and then the cytotoxic activity of NK cells was analyzed by coincubation with MDA-MB231 cells $(E: T$ ratio=10:1) for 4 hrs. NK cell-induced cytotoxic damage to MDAMB231 cells was analyzed by CFSE/7-AAD staining. (C) XRP44X-treated time-dependent activation of NK-92Ml cells was analyzed by a CytoTox $\mathrm{Glo}^{\mathrm{TM}}$ cytotoxicity assay. The error bars represent the standard errors from three independent experiments, which were each performed using triplicate samples. ${ }^{* * *} p<0.005$ (Student's $t$-test). Values labeled with different letters are significantly different from one another ( $p<0.01$, ANOVA test). E:T, effector:target. 
A

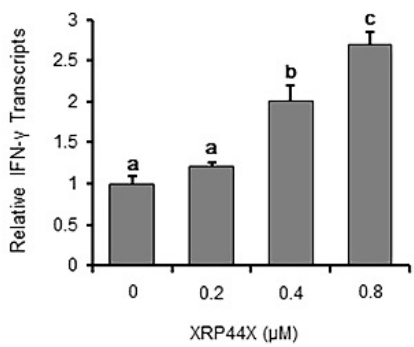

B

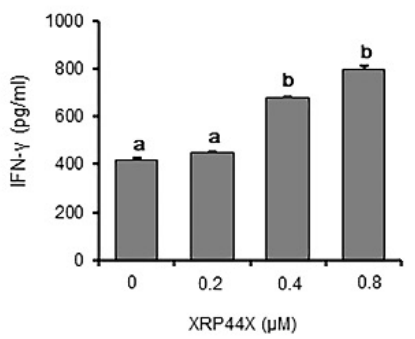

C

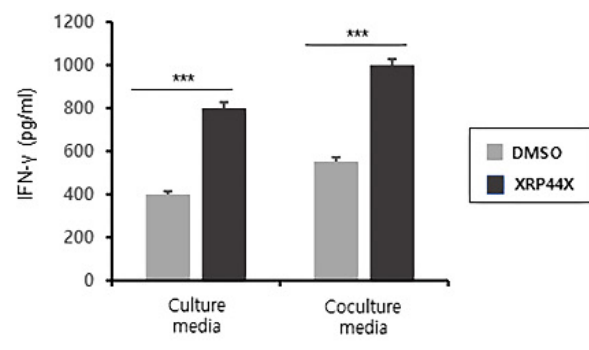

Fig. 3. XRP44X increases IFN- expression in NK-92MI cells. (A) The effect of XRP44X on the transcription of IFN-y was quantitatively analyzed by treating NK-92Ml cells with the indicated concentration of XRP44X for $48 \mathrm{hrs}$ and analyzing IFN-y expression by qRT-PCR. (B) The effect of XRP44X on the secretion of IFN- was analyzed by treating NK-92MI cells with the indicated concentration of XRP44X for 48 hrs and analyzing the culture media by ELISA. (C) The effect of XRP44X on the secretion of IFN- $y$ in the presence of target MDA-MB231 cells was analyzed by treating with NK-92MI cells with the indicated concentration of XRP44X for $48 \mathrm{hrs,} \mathrm{coculturing} \mathrm{with} \mathrm{the} \mathrm{target} \mathrm{cells} \mathrm{and} \mathrm{analyzing} \mathrm{the} \mathrm{culture} \mathrm{media} \mathrm{by} \mathrm{ELISA.} \mathrm{XRP44X-treated} \mathrm{NK-92MI}$ cells were cocultured with MDA-MB231 cells for $4 \mathrm{hrs}$. The error bars represent the standard errors from three independent experiments, which were each performed using triplicate samples. ${ }^{* \star} p<0.005$ (Student's $t$-test). Values labeled with different letters are significantly different from one another $(p<0.01$, ANOVA test).

following XRP44X treatment. As shown in Fig. 4A, JNK was significantly phosphorylated by 0.8 $\mu \mathrm{M}$ XRP44X, whereas $\mathrm{p} 38$ and ERK1/2 were not phosphorylated under the same conditions. To determine whether activation of JNK by XRP44X is associated with NK activation, we examined the effect of chemical inhibition of JNK phosphorylation on XRP44X-mediated activation of NK cells. As expected, the degree of NK activation by XRP44X was significantly decreased when NK cells were cotreated with the JNK inhibitor SP600125 to inhibit XRP44X-mediated phosphorylation of JNK (Fig. 4B-C).

Finally, we examined whether XRP44X-mediated activation of NK cells sustains their enhanced activity in the absence of XRP44X. For this purpose, we first cultured NK-92MI cells for $48 \mathrm{hr}$ in the presence of XRP44X and then transferred the cells to fresh media without XRP44X. As shown in Fig. 4D, NK cells that were activated by XRP44X maintained their enhanced cytotoxic activity against MDA-MB231 cells up to three days after the removal of XRP44X. Taken together, we concluded that XRP44X activates naive NK cells in the absence of target cells and that XRP44Xmediated activation of JNK signaling is one of the major mechanisms that explains the enhanced activity of NK cells.

\section{DISCUSSION}

The ETS family transcription factor ETS1 regulates the expression of signaling molecules that are essential for NK cell activation, and $E t s 1^{-/} \mathrm{NK}$ cells are in a chronically activated state (Ramirez et al., 2012). Based on this report, we examined whether ELK3, another ETS family transcription factor, is associated with the cytotoxic activity of NK cells. The pyrazole chemical XRP44X was identified as a candidate anticancer drug that inhibits ELK3 activity in various cells, including cancer cells (Wasylyk et al., 2008; Chen et al., 2012; Semenchenko et al., 2016), and we applied XRP44X to NK cells.

Pyrazole is a type of chemical that has a 5-membered ring structure with 2 nitrogen atoms and aromatic heterocycles. Its derivatives have been applied for a wide range of purposes, including pesticidal, antibacterial, antihepatotoxicity, anti-inflammatory, and analgesic activities. We confirmed in this study that the pyrazole derivative compound XRP44X increases the cell-mediated cytotoxic ability of NK cells. XRP44X is an inhibitor of ELK3, the RAS sub-signal molecule (Semenchenko 
A

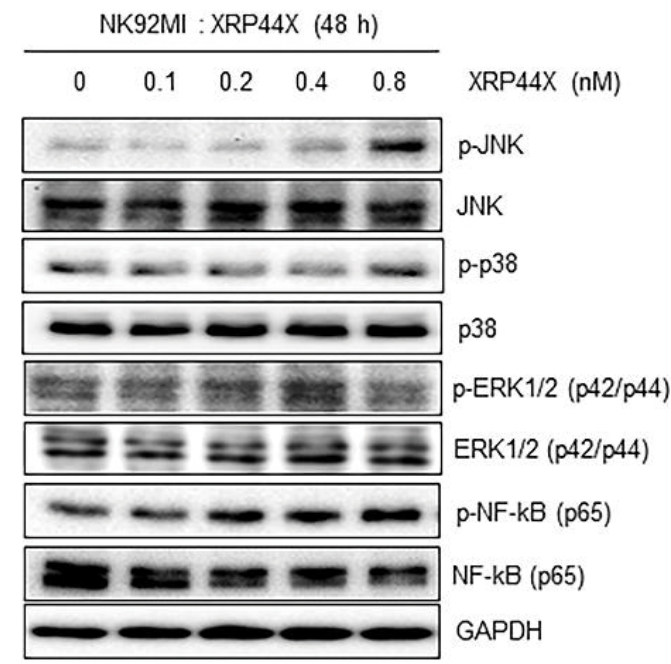

C

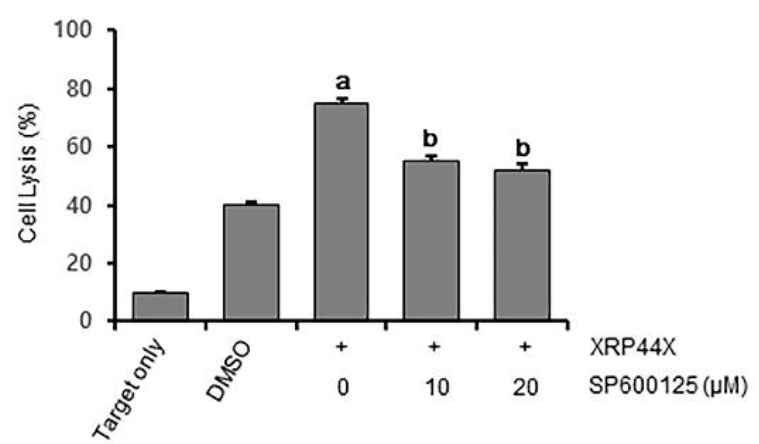

B

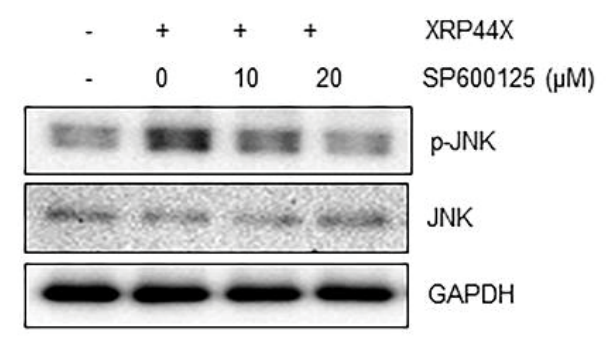

D

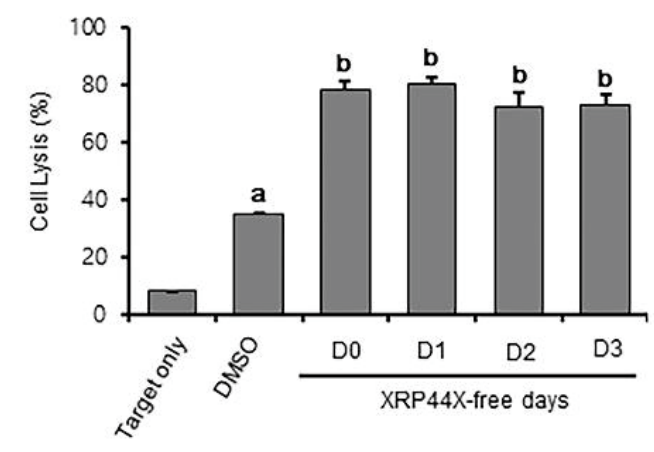

Fig. 4. XRP44X enhances the cytotoxic activity of NK-92MI cells by activating the JNK signaling pathway. (A) The effect of XRP44X on the activation of the indicated signaling pathway in NK-92MI cells. NK cells were treated with XRP44X for $48 \mathrm{hrs}$ and then analyzed for the expression of the indicated proteins by immunoblotting. (B) The indicated concentration of JNK inhibitor (SP600125) was coadministered with $0.8 \mu \mathrm{M}$ XRP44X for 48 hrs, and then the phosphorylation of JNK (p-JNK) was analyzed by immunoblotting. (C) Cytotoxicity of NK-92Ml cells treated with the indicated concentration of JNK inhibitor (SP600125) and $0.8 \mu \mathrm{M}$ XRP44X for $48 \mathrm{hrs}$ was analyzed by a CytoTox-Glo ${ }^{\mathrm{TM}}$ cytotoxicity assay. NK-92-MI cells were coincubated with MDAMB231 cells $(E: T$ ratio=10:1) for 4 hrs. (D) NK-92Ml cells were cultured in the presence of $0.8 \mu M$ XRP44X for 48 hrs and then further cultured in the absence of XRP44X for the indicated days (XRP44X-free days). The cytotoxicity of NK-92Ml cells was analyzed by a CytoTox-Glo ${ }^{\mathrm{TM}}$ cytotoxicity assay. NK-92-MI cells were coincubated with MDA-MB231 cells (E:T ratio=10:1) for 4 hrs. The error bars represent the standard errors from three independent experiments, which were each performed using triplicate samples. Values labeled with different letters are significantly different from one another $(p<0.01$, ANOVA test). JNK, c-JUN N-terminal kinase. E:T, effector:target.

et al., 2016), and a microtubule depolymerizing reagent (Wasylyk et al., 2008). When we treated the triple negative breast cancer cell line MDA-MB231 with XRP44X, we observed that the cells were condensed and could not maintain their structure (data not shown). This is thought to be the result of XRP44X affecting the cell structure of MDA-MB231 cells.

A recent study of pyrazole compounds on NK cells showed that 4-methylpyrazole (4-MP) activates NK cells (Yi et al., 2015) (1). This finding reveals that 4-MP stimulates NK cells to express IFN $-\gamma$, and in our results, a more complex pyrazole, XRP44X, also promoted the expression of IFN- $\gamma$ at the transcript level. From these studies, we hypothesize that there is a correlation between pyrazoles and IFN- $\gamma$ expression in NK cells. Usually, IFN- $\gamma$ is produced when NK cells recognize target cells (Rajagopalan et al., 2001). Our study revealed that XRP44X treatment induces increases of IFN- $\gamma$ production at resting state of NK cells without target cell contact. 
Since RNA level of IFN- $\gamma$ is increased by XRP44X, it is estimated that transcriptional activation of cytotoxic molecules such as IFN- $\gamma$, possibly through the associated signaling pathway, might be one of the main mechanism of XRP44X to enhance anticancer activity of NK cells.

The JNK (c-Jun N-terminal kinase) pathway plays an important role in NK cell activation. There have been reports that the JNK pathway is required for granule depolarization ( $\mathrm{Li}$ et al., 2008) or is involved in the constitutive expression of CCL5 (Kumar et al., 2009). XRP44X affects various signaling pathways in NK cells, but it is particularly characterized by changes in the phosphorylation of JNK, which is thought to be a factor in increasing NK cell-mediated cytotoxicity. We showed an interesting result regarding the time course activation of JNK and NF$\kappa \mathrm{B}$. The phosphorylation of JNK dramatically increased with XRP44X treatment and decreased rapidly after XRP44X was removed. On the other hand, phosphorylation of NF- $\mathrm{KB}$ increased slowly until the next day and then decreased slowly. This kind of crosstalk between signaling pathways may be an important result for understanding the activation mechanism of NK cells. Since XRP44X was originally identified as an anticancer agent that depolymerized microtubules of cancer cells, our finding that XRP44X enhances the cytotoxic activity of NK-92MI cells presents a new paradigm for the combination of chemotherapy and immunotherapy in tumor treatment.

\section{REFERENCES}

Bauer S, Groh V, Wu J, Steinle A, Phillips JH, Lanier LL, Spies T (1999) Activation of NK cells and $\mathrm{T}$ cells by NKG2D, a receptor for stress-inducible MICA. Science 285:727-729.

Chen J, Sun WL, Wasylyk B, Wang YP, Zheng H (2012) c-Jun N-terminal kinase mediates microtubule-depolymerizing agent-induced microtubule depolymerization and $\mathrm{G} 2 / \mathrm{M}$ arrest in MCF-7 breast cancer cells. Anticancer Drugs 23:98-107.

Ge H, Lv X, Jin H, Tian Z, Li Y, He H (2017) The role of endovascular treatment in unruptured basilar tip aneurysms. Interv Neuroradiol 23:8-13.

Huntington ND (2014) The unconventional expression of IL-15 and its role in NK cell homeostasis. Immunol Cell Biol 92:210-213.

Janeway CA, Medzhitov R (2002) Innate immune recognition. Annu Rev Immunol 20:197-216.

Kong SY, Kim KS, Kim J, Kim MK, Lee KH, Lee JY, Oh N, Park JI, Park JH, Heo SH, Shim SH, Lee DR, Kim KP, Park KS (2016) The ELK3-GATA3 axis orchestrates invasion and metastasis of breast cancer cells in vitro and in vivo. Oncotarget 7:65137-65146.

Kumar D, Hosse J, von Toerne C, Noessner E, Nelson PJ (2009) JNK MAPK pathway regulates constitutive transcription of CCL5 by human NK cells through SP1. J immunol 182:10111020.

Lee JH, Hur W, Hong SW, Kim JH, Kim SM, Lee EB, Yoon SK (2017) ELK3 promotes the migration and invasion of liver cancer stem cells by targeting HIF-1 $\alpha$. Oncol Rep 37:813822.

Li C, Ge B, Nicotra M, Stern JN, Kopcow HD, Chen X, Strominger JL (2008) JNK MAP kinase activation is required for MTOC and granule polarization in NKG2D-mediated NK cell cytotoxicity. Proc Natl Acad Sci USA 105:3017-3022.

Rajagopalan S, Fu J, Long EO (2001) Cutting Edge: Induction of IFN- $\gamma$ production but not cytotoxicity by the killer cell Ig-like receptor KIR2DL4 (CD158d) in resting NK cells. J Immunol 167:1877-1881.

Ramirez K, Chandler KJ, Spaulding C, Zandi S, Sigvardsson M, Graves BJ, Kee BL (2012) Gene deregulation and chronic activation in natural killer cells deficient in the transcription factor ETS1. Immunity 36:921-932. 
Rana K, Whalen M (2015) Activation of protein kinase C and protein kinase D in human natural killer cells: effects of tributyltin, dibutyltin, and tetrabromobisphenol A. Toxicol Mech Methods 25:680-688.

Romera-Cárdenas G, Thomas LM, Lopez-Cobo S, García-Cuesta EM, Long EO, Reyburn HT (2016) Ionomycin treatment renders NK cells hyporesponsive. PLoS One 11:e0150998

Semenchenko K, Wasylyk C, Cheung H, Tourrette Y, Maas P, Schalken JA, van der Pluijm G, Wasylyk B (2016) XRP44X, an inhibitor of Ras/Erk activation of the transcription factor Elk3, inhibits tumour growth and metastasis in mice. PLoS One 11:e0159531.

Sim GC, Radvanyi L (2014) The IL-2 cytokine family in cancer immunotherapy. Cytokine Growth Factor Rev 25:377-390.

Sutherland CL, Chalupny NJ, Schooley K, VandenBos T, Kubin M, Cosman D (2002) UL16binding proteins, novel MHC class I-related proteins, bind to NKG2D and activate multiple signaling pathways in primary NK cells. J Immunol 168:671-679.

Wasylyk C, Zheng H, Castell C, Debussche L, Multon MC, Wasylyk B (2008) Inhibition of the Ras-Net (Elk-3) pathway by a novel pyrazole that affects microtubules. Cancer Res 68:12751283.

Yi HS, Eun HS, Lee YS, Jung JY, Park SH, Park KG, Choi HS, Suh JM, Jeong WI (2015) Treatment with 4-methylpyrazole modulated stellate cells and natural killer cells and ameliorated liver fibrosis in mice. PLoS One 10:e0127946. 
